\title{
Applying Tai Chi as a rehabilitation program for stroke patients in the recovery phase: study protocol for a randomized controlled trial
}

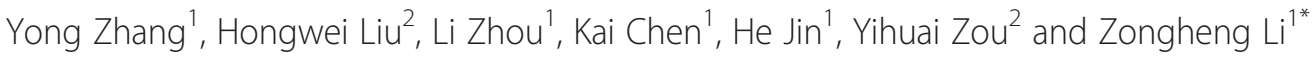

\begin{abstract}
Background: As the second commonest cause of death and a major cause of disability worldwide, stroke has greatly influenced patients' quality of life and created a huge public health burden. As a special form of physical activity that has been widely practiced in China, and even throughout the world, Tai Chi may be favorable for the rehabilitation of stroke patients. Several studies have been conducted to investigate the rehabilitative effects of Tai Chi for stroke patients, but none of them have been focused on the recovery phase (2 to 24 weeks) of stroke.

Methods/design: This study is an assessor-blinded randomized controlled trial. A total of 50 eligible participants will be randomly assigned to either a control group or a Tai Chi group. Patients in the control group will receive standard, conventional rehabilitation therapies, and a combination of Tai Chi and conventional rehabilitation programs will be applied in the Tai Chi group. The recovery of motor impairment, functional activity and balance abilities as measured with the Fugl-Meyer Assessment, Barthel Index and Berg Balance Scale will be assessed as primary outcome measures. The secondary outcome measures to be used are the scores on the Stroke-Specific Quality of Life Scale, the National Institutes of Health Stroke Scale and the objective parameters of the RSscan footscan gait system. All assessments will be conducted at baseline, 4 weeks after the rehabilitation course and at the end of 3-month follow-up.
\end{abstract}

Discussion: The results of this study will provide preliminary evidence regarding the efficacy and feasibility of Tai Chi as an additional rehabilitative program for stroke patients in the recovery phase.

Trial registration: Chinese Clinical Trial Register ID: ChiCTR-TRC-13003661 (7 October 2013)

Keywords: Recovery phase, Rehabilitation, Stroke, Tai Chi

\section{Background}

Stroke has become the second commonest cause of death and a major cause of disability worldwide [1]. It has greatly influenced stroke patients' quality of life and created a huge public health burden [2]. With the population aging and lifestyles changing, the burden will increase greatly in the next 20 years, especially in the developing countries [3]. Recent epidemiological studies have shown that there are over 7 million stroke survivors living in China, and about $70 \%$ of them have functional disabilities

\footnotetext{
* Correspondence: lee_zongheng@163.com

'Department of Rehabilitation, Dongzhimen Hospital affiliated to Beijing University of Chinese Medicine, No. 5, Haiyuncang, Dongcheng District, Beijing 100700, China

Full list of author information is available at the end of the article
}

[4]. This reality drove us to search for effective modalities of treatment for stroke rehabilitation.

Tai Chi (also called Taiji or Tai Chi Chuan) is a famous intangible cultural heritage that originated in China as a martial art hundreds of years ago [5]. Built upon the mind-body connection, Tai Chi combines physical movement, meditation and breathing to induce relaxation and tranquility of the mind, and it improves balance, postural control, movement coordination, strength and flexibility [6]. In the past decade, a substantial number of studies and reviews have been conducted in the field of the clinical use of Tai Chi [7-11]. Recently, the significant effects of Tai Chi for fibromyalgia and Parkinson's disease rehabilitation have been confirmed, and related studies published in the New England Journal of 
Medicine have brought great attention to and general agreement on the clinical effects of Tai Chi [12,13].

Physical activity is an important component of comprehensive stroke rehabilitation programs implemented to reduce disabilities. As a special form of physical activity that has been widely practiced in China, and even throughout the world, Tai Chi may be favorable for the rehabilitation of stroke patients $[9,14,15]$. Tai Chi is considered a complex, multicomponent intervention that integrates physical, psychosocial, emotional, spiritual and behavioral elements [12]. The main essence of Tai Chi practice is similar to Bobath therapy and proprioceptive neuromuscular facilitation techniques, which made it possible for us to apply Tai Chi for stroke rehabilitation [9].

Several studies have been conducted to explore the health-enhancing qualities of Tai Chi for stroke patients. The studies conducted by Taylor et al. demonstrated that community-based Tai Chi practice was a safe and feasible program for stroke patients [16,17]. Another study indicated that a 12-posture short-form Tai Chi exercise was helpful in enhancing the standing balance of patients with a stroke history longer than 6 months after onset [18]. To the best of our knowledge, there has been no study to date in which Tai Chi was used in a rehabilitation program for stroke patients in the recovery phase (2 to 24 weeks). In this study protocol article, we will describe the rationale, design and analytic methods of a randomized controlled trial designed to investigate the rehabilitative effects of Tai Chi among hospitalized stroke patients in the recovery phase. We hypothesized that patients in the Tai Chi group would demonstrate better improvement compared with that in the control group.

\section{Methods/design \\ Study design and setting}

The study will be conducted from 1 January 2014 to 31 December 2014 in Dongzhimen Hospital, which is affiliated with the Beijing University of Chinese Medicine. All participants will be allocated in a 1:1 ratio into either a control group or a Tai Chi group. Patients in the control group will receive standard conventional rehabilitation therapies, and a combination of Tai Chi and conventional rehabilitation therapies will be applied in the Tai Chi group. The participants' scores on the Fugl-Meyer Assessment, Barthel Index and Berg Balance Scale will be assessed as primary outcome measures. The secondary outcome measures will be scores on the StrokeSpecific Quality of Life Scale, the National Institutes of Health Stroke Scale and the objective parameters of the RSscan footscan gait system (RSscan International, Olen, Belgium). All assessments will be conducted at baseline, 4 weeks after the rehabilitation course and at the end of 3-month follow-up. The study design is summarized in Figure 1.

\section{Ethics}

The study protocol was approved by the research ethics committee of Dongzhimen Hospital, which is affiliated with the Beijing University of Chinese Medicine (no. 2013JS201), and it follows the principles of the CONSORT statements as well as the Declaration of Helsinki. The trial is registered with the Chinese Clinical Trial Register (ChiCTR-TRC-13003661). The research ethics committee will also be in charge of supervising all procedures carried out in our study, including patient recruitment, randomization, conduct of the study and data storage. In cases of changes to our study protocol, we have to hand in a written application to the research ethics committee. The committee members will then decide whether it is necessary to change the study protocol.

\section{Randomization and allocation concealment}

This study is designed as a pilot study in which 20 participants will be included in each group. Allowing for a $25 \%$ dropout rate, we will recruit 25 patients in each group which means a total of 50 patients should be included during the whole trial $[19,20]$. Participants will be assigned in a 1:1 ratio according to a computer-generated randomization list. Assignments will be sealed in opaque envelopes and will be opened by the study staff following informed consent procedures and baseline testing. The participants and researchers will know the allocated group, but the outcome assessors and data analysts will not.

\section{Informed consent}

Prior to the study, the general study process and the responsibilities of both participants and researchers will be explained to potential participants. They will be told that their entry into the trial is entirely voluntary and that they can withdraw at any time. In the event of their withdrawal, the data collected cannot be erased and will be used in the final analyses. Written informed consent should be obtained from each participant before any interventions related to the study are started. ZL will be responsible for obtaining informed consent from all participants.

\section{Inclusion criteria}

Participants meeting the following criteria will be included:

1. Stroke that has been confirmed by computed tomography or magnetic resonance imaging

2. Between 40 and 75 years old

3. First episode of stroke or a history of stroke, but no serious deformity, and a modified Rankin Scale grade $\leq 2$ 


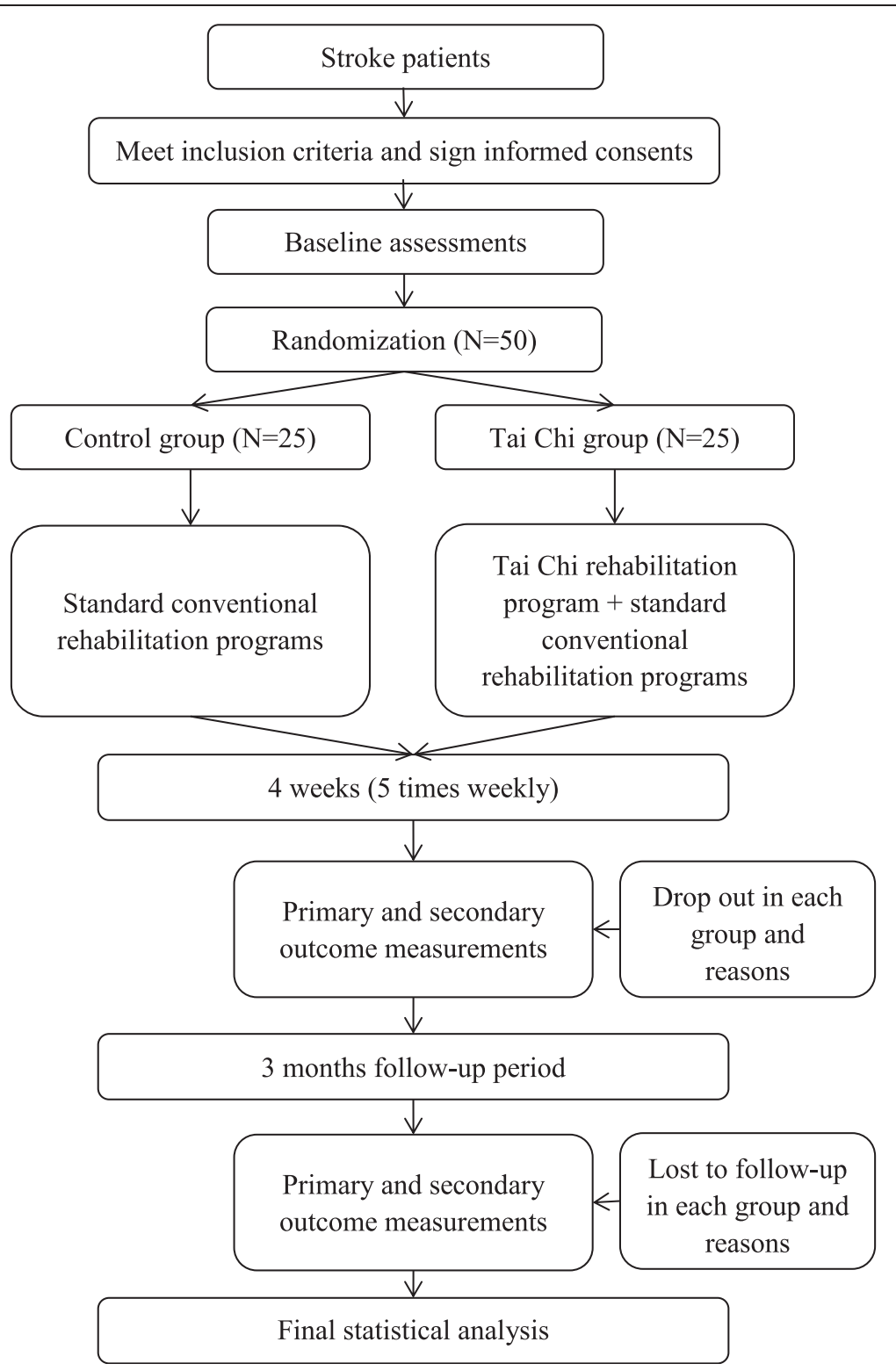

Figure 1 Flowchart of the study design.

4. Between 2 and 20 weeks after the onset of the current stroke

5. Unilateral lower-extremity hemiplegia and able to walk $\geq 6$ meters [18]

6. Blood pressure $<160 / 100 \mathrm{mmHg}$

7. Sufficient cognition to follow commands, Mini Mental State Examination score $>24$

8. No experience with Tai Chi

9. Voluntary participation in the trial and provision of informed consent

\section{Exclusion criteria}

Participants meeting the following criteria will be excluded:
1. Received thrombolytic therapy or surgery

2. Vital signs unstable or worsening condition

3. Severe primary diseases of the cardiovascular system, liver, kidney or hematopoietic system

4. Pregnant or lactating women

5. Participation in other clinical trials

\section{Interventions}

\section{Control group}

Patients who are assigned to the control group will be engaged in standard conventional rehabilitation programs that consist of individualized amounts of various therapy options for stroke patients (for example, Bobath therapy or proprioceptive neuromuscular facilitation) 
provided by occupational and physical therapists. The conventional rehabilitation program consists of a series of standard rehabilitation therapies for every stroke symptom. We will select certain therapies for each of the patients according to their symptoms. The standard rehabilitation program will be carried out five times per week (that is, Monday to Friday) for 4 weeks, and each time the rehabilitation course will last for approximately 1 hour. All conventional rehabilitation programs will be conducted by two qualified therapists ( $\mathrm{YZ}$ and $\mathrm{HJ}$ ).

\section{Tai Chi group}

To study the additional effects of Tai Chi to the standard conventional rehabilitation programs, patients allocated to the intervention group will be engaged in Tai Chi rehabilitation programs in addition to the control therapies.

The Tai Chi rehabilitation program is specially established for stroke patients with lower-extremity hemiplegia by an expert panel comprising a qualified Tai Chi instructor, an experienced therapist from the Department of Rehabilitation at Dongzhimen Hospital and a senior doctor from the hospital's stroke center. The Tai Chi rehabilitation program consists of six Tai Chi movements listed in order of difficulty. The six Tai Chi movements are Commencing, Part the Wild Horse's Mane, Wave Hands Like Clouds, Brush Knee and Twist Step, Reverse Reeling Forearm and Closing derived from the 24 forms of simplified Tai Chi recommended as a popular health sport by the General Administration of Sport of China [7].

Our goal of selecting such movements to design a Tai Chi rehabilitation program is to help stroke patients with lower-extremity hemiplegia to maintain motor function and postural balance control. Thus, in the actual practice of the Tai Chi rehabilitation program, we will not focus on the continuity between different movements, but will put much emphasis on the accuracy and quality of performing every single movement. Each movement will be integrated therapeutically, especially for the stroke-affected side, by performing symmetrical and coordinated movements such as trunk rotation and weight-bearing, controlled and coordinated displacement of the body's center of mass, and rebuilding of the affected ankle and knee joints by anteroposterior and mediolateral stepping [13].

The Tai Chi rehabilitation program will take place five times per week (from Monday to Friday) for 4 weeks, and each session will include a warm-up for 10 minutes, specified Tai Chi exercises performed for 40 minutes and cooling down for 10 minutes. The Tai Chi rehabilitation program will be applied 20 to 30 minutes after the patients finish the conventional rehabilitation programs. An experienced Tai Chi instructor who has been qualified and engaged in the teaching of Tai Chi courses for 10 years will lead the Tai Chi rehabilitation program. In the first week of the program, we will emphasize primarily learning and practicing single forms of Tai Chi exercises with multiple repetitions. Instruction will cover learning new forms and reviewing and practicing forms learned in previous sessions. For the remaining 3 weeks, the focus will be on performing individual forms to enhance motor function recovery and postural balance. Natural breathing will also be emphasized as part of the Tai Chi rehabilitation program. Patients will be told to breathe as they usually do so that they can focus more on the Tai Chi movements and achieve better functional recovery.

\section{Follow-up}

After the 4-week supervised rehabilitation courses in the hospital, all patients will be discharged from the hospital and begin an additional 3-month unsupervised followup period. Owing to the specificity of stroke, patients are always encouraged to attend community-based rehabilitation courses. For patients in both of our study groups, we will encourage them to attend such standard rehabilitation courses. Meanwhile, patients in the Tai Chi group will be encouraged to maintain their Tai Chi practice, and they will be asked to fill out forms to record the times and durations of their Tai Chi practice. Patients in the control group will be asked to fill out similar forms to record their rehabilitation program attendance. All forms will be returned to the researchers for monitoring at the end of the follow-up period.

\section{Outcome measures}

Data collection will be performed by a trained, certified assessor who will be blinded to patients' group assignment at baseline, after the intervention (4 weeks) and at the end of follow-up (3 months).

\section{Basic characteristics variables}

Demographic information of the participants will be collected at baseline to describe the sample, compare conditions and investigate characteristics associated with outcomes. These measures will include participants' sex, age, ethnicity, marital status, educational background, time since the attack of stroke, use of medication and other details. Vital signs such as respiration rate, pulse rate, resting blood pressure and body temperature will be measured by nurses.

\section{Primary outcomes}

The first primary outcome measurement is the recovery of motor impairment. We will apply the Fugl-Meyer Assessment (FMA) to measure it. Developed as the first quantitative evaluative instrument for measuring sensorimotor 
stroke recovery, the FMA is a well-designed, feasible and efficient clinical examination method that has been tested widely in the stroke population across different stroke recovery time points [21]. The FMA motor scale is recommended highly as a clinical and research tool for evaluating changes in motor impairment following stroke [22]. Its primary value is the 100-point motor domain, which has received the most extensive evaluation. It includes an assessment of the upper extremity and the lower extremity. In the present study, we will focus on the measurement of the lower extremity, which includes two subsections-the leg and coordination-with a total possible score of 34 .

The improvements in activities of daily living that can be measured with the Barthel Index (BI) will be listed as another primary outcome measurement. The BI is a frequently used functional outcome measure of activities of daily living in stroke trials [23]. It is also widely used in clinical practice to assess baseline abilities, to quantify functional changes after rehabilitation and to inform discharge planning. The scale describes ten task items and is scored according to the amount of time or assistance required by the patient. These ten items are feeding, bathing, grooming, dressing, continence of bowels and bladder, transferring to and from a toilet, moving from wheelchair to bed and return, walking on a level surface for 45 meters and going up and down stairs. The total score ranges from 0 to 100 , with lower scores representing greater nursing dependency [24].

The static and dynamic balance abilities will also be applied as primary outcome measurements. The Berg Balance Scale (BBS) is a clinical test widely used to quantitatively assess the static and dynamic balance abilities of older adults [25]. It comprises a set of 14 simple balance-related tasks, ranging from standing up from a sitting position to standing on one foot. The total score ranges from 0 to 56 , with 0 to 20 corresponding to a high fall risk, 21 to 40 to a medium fall risk and 41 to 56 to a low fall risk. The BBS has been widely used to assess balance abilities in recent studies of the clinical effects of Tai Chi in therapy for various diseases, including stroke $[18,26]$. It is identified as the most commonly used assessment tool across the continuum from acute care to community-based rehabilitation in stroke. The authors of a recent systematic review suggested that the BBS provides an effective and appropriate assessment of balance in patients with stroke and should be considered for use in measuring outcomes of various stroke rehabilitation interventions [27].

\section{Secondary outcomes}

To the best of our knowledge, only scale evaluations have been used to measure the outcomes of stroke rehabilitation in most previous studies. Objective outcome measurements have been used in few of them. In the present study, we will try to measure several objective parameters by using the RSscan footscan gait system (2096 mm $\times 469 \mathrm{~mm} \times 18 \mathrm{~mm}, 16,384$ sensors, $480 \mathrm{~Hz}$, RSscan International, Olen, Belgium). Consisting of a sensor array, a data collector, a walkway and a computer, the footscan gait system is sensitive in detecting the parameters of plantar pressure data and time phase when subjects are standing or walking on the plate. It is designed to test the characteristics of different kinds of movement, especially among athletes [28]. Nowadays, it is also used in clinical studies to provide quantitative assessments $[29,30]$. In the present study, patients will be asked to stand and walk on the plate to become familiar with the system before formal data are collected. Data of plantar force distribution, impulse, maximum force, maximum pressure and time of walk phase will be collected to evaluate lower-extremity function.

We will also assess the quality of life in both groups as secondary outcomes. The Stroke-Specific Quality of Life Scale (SS-QOL) is a patient-reported outcome measure intended to provide an assessment of health-related quality of life specific to patients with stroke [31]. The SS-QOL questionnaire consists of 49 items in the 12 domains of energy, family roles, language, mobility, mood, personality, self-care, social roles, thinking, upperextremity function, vision and work/productivity. Scoring of the SS-QOL is rated on a 5-point Likert scale. The domains are scored separately, and a total score is also calculated, with higher scores indicating better function.

The National Institutes of Health Stroke Scale (NIHSS) is a widely used, validated tool used in nearly all large clinical stroke trials to document baseline and outcome severity of neurological impairment $[32,33]$. The NIHSS comprises tests of 11 items, including the levels of consciousness, selected cranial nerves, motor function, sensory function, cerebellar function, language and inattention (that is, neglect). Total scores range from 0 to 42 , with scores $>25$ indicating very severe neurological impairment, scores of 5 to 24 suggesting moderately severe to severe impairment and scores $<5$ indicating mild impairment.

\section{Adverse events}

All unexpected adverse events during the intervention period will be reported, and the causality related to the Tai Chi intervention will be analyzed. If any adverse event occurs, the Tai Chi instructor or study managers will provide the corresponding treatment to the participant. The adverse events will be immediately reported to the primary investigator and ethics committee to decide if the participant needs to withdraw from the trial. In case of stroke recurrence or other worsening conditions, patients will be stopped from participating in the study and will be referred for further treatment. 


\section{Statistical analysis}

Statistical analysis will be conducted by statisticians who are independent from the research team using SPSS 12.0 software for Windows (IBM SPSS, Chicago, IL, USA). The categorical variables will be presented with frequencies or percentages, and the continuous variables will be presented as means and standard deviations. Demographic and clinical assessments of two groups will be compared upon admission to address the baseline characteristics. For both groups, data of primary and secondary outcome measurements collected after 4 weeks and at the end of 3 months will be compared with baseline data, respectively, to evaluate the rehabilitative effects within groups. To evaluate the effects of Tai Chi on stroke rehabilitation, data of all outcome measurements after 4 weeks and at the end of 3 months will be compared between the two groups. Unpaired two-sample $t$-tests (continuous data) and $X^{2}$ analysis (categorical data) will be applied in data analyses between two groups. Nonparametric methods will be used when assumptions of normality are violated. Patients with missing data before 4 weeks will be eliminated. We will also conduct an intention-to-treat analysis if participants are lost to follow-up at 3 months. The statistical significance threshold will be set at 0.05 (two-sided), with $95 \%$ confidence intervals.

\section{Discussion}

The results of this study will provide preliminary evidence regarding the efficacy and feasibility of Tai Chi as an additional rehabilitative intervention for stroke patients in the recovery phase. Furthermore, if the results are positive, this study will contribute to the establishment of further guidance in applying Tai Chi rehabilitation programs during the early stages of stroke.

The studies conducted by Taylor et al. and Au-Yeung et al. also applied Tai Chi rehabilitation programs for stroke patients, and their results indicated that Tai Chi was helpful [16-18]. However, in these previous studies, Tai Chi practice was geared mainly for the sequelae stage rather than the recovery phase of stroke. As recent studies have suggested, stroke rehabilitation should be started as soon as the patient's vital signs become stable $[34,35]$. Thus, it would be more reasonable and closer to clinical reality to apply Tai Chi for stroke rehabilitation in the early stages of stroke.

In most previous studies, researchers applied only scale evaluations to measure the outcomes of stroke rehabilitation. Few of them have introduced objective outcome measurements. In the present study, by using the footscan gait system, we will measure several objective parameters, including the plantar force distribution, impulse, maximum force, maximum pressure and time of walk phase, which will provide objective evaluation of the effects of different rehabilitation programs on lowerextremity function.

One limitation concerns the fact is that this is only a preliminary single-center study. Another limitation is that the therapist is not blinded in the present trial. A blinded study involving Tai Chi is challenging to conduct because it is almost impossible to blind the therapist and patients.

\section{Trial status}

This trial is currently ongoing and 6 participants are still in need to complete the whole study.

\section{Abbreviations}

BBS: Berg Balance Scale; BI: Barthel Index; FMA: Fugl-Meyer Assessment; NIHSS: National Institutes of Health Stroke Scale; SS-QOL: Stroke-Specific Quality of Life Scale.

\section{Competing interests}

The authors declare that they have no competing interests.

\section{Authors' contributions}

All authors participated in the conception and design of the initial protocol. $Y Z$ drafted the manuscript. ZHL and $Y H Z$ revised the manuscript. ZHL is the Tai Chi instructor. HWL is in charge of patient recruitment. LZ is responsible for outcome measurement. KC is the operator of the footscan gait system. YZ and HJ will carry out the rehabilitation programs. All authors read and approved the final manuscript.

\section{Acknowledgements}

The study is supported by the Beijing Capital Characteristic Clinical Application Research Project from Beijing Municipal Science \& Technology Commission of China (grant Z141107002514145) and the Middle-aged Teachers Research Funds of Beijing University of Chinese Medicine (grant 2013-JYBZZ-JS-201).

\section{Author details}

${ }^{1}$ Department of Rehabilitation, Dongzhimen Hospital affiliated to Beijing University of Chinese Medicine, No. 5, Haiyuncang, Dongcheng District, Beijing 100700, China. '2Department of Neurology and Stroke Center, Dongzhimen Hospital affiliated to Beijing University of Chinese Medicine, No. 5, Haiyuncang, Dongcheng District, Beijing 100700, China.

Received: 5 June 2014 Accepted: 24 November 2014

Published: 11 December 2014

\section{References}

1. Johnston SC, Mendis S, Mathers CD: Global variation in stroke burden and mortality: estimates from monitoring, surveillance, and modelling. Lancet Neurol 2009, 8:345-354.

2. Feigin VL, Forouzanfar MH, Krishnamurthi R, Mensah GA, Connor M, Bennett DA, Moran AE, Sacco RL, Anderson L, Truelsen T, O'Donnell M, Venketasubramanian N, Barker-Collo S, Lawes CM, Wang W, Shinohara Y, Witt E, Ezzati M, Naghavi M, Murray C, the Global Burden of Diseases, Injuries, and Risk Factors Study 2010 (GBD 2010) and the GBD Stroke Experts Group: Global and regional burden of stroke during 1990-2010: findings from the Global Burden of Disease Study 2010. Lancet 2014, 383:245-255. A published erratum appears in Lancet 2014, 383:218.

3. Giroud $M$, Jacquin $A$, Béjot $Y$ : The worldwide landscape of stroke in the 21st century. Lancet 2014, 383:195-197. A published erratum appears in Lancet 2014, 383:696.

4. Liu M, Wu B, Wang WZ, Lee LM, Zhang SH, Kong LZ: Stroke in China: epidemiology, prevention, and management strategies. Lancet Neurol 2007, 6:456-464.

5. Wang J, Feng B, Yang XC, Liu W, Teng F, Li SJ, Xiong XJ: Tai Chi for essential hypertension. Evid Based Complement Alternat Med 2013, 2013:215254. 
6. Zhang L, Layne C, Lowder T, Liu J: A review focused on the psychological effectiveness of Tai Chi on different populations. Evid Based Complement Alternat Med 2012, 2012:678107.

7. Zheng G, Lan X, Li M, Ling K, Lin H, Chen L, Tao J, Li J, Zheng X, Chen B, Fang $Q$ : The effectiveness of Tai Chi on the physical and psychological wellbeing of college students: a study protocol for a randomized controlled trial. Trials 2014, 15:129.

8. Wayne PM, Kiel DP, Buring JE, Connors EM, Bonato P, Yeh GY, Cohen CJ, Mancinelli C, Davis RB: Impact of Tai Chi exercise on multiple fracturerelated risk factors in post-menopausal osteopenic women: a pilot pragmatic, randomized trial. BMC Complement Altern Med 2012, 12:7.

9. Tousignant M, Corriveau H, Kairy D, Berg K, Dubois MF, Gosselin S, Swartz RH, Boulanger JM, Danells C: Tai Chi-based exercise program provided via telerehabilitation compared to home visits in a post-stroke population who have returned home without intensive rehabilitation: study protocol for a randomized, non-inferiority clinical trial. Trials 2014, 15:42.

10. Winters-Stone KM, Li F, Horak F, Luoh SW, Bennett JA, Nail L, Dieckmann N: Comparison of tai chi vs. strength training for fall prevention among female cancer survivors: study protocol for the GET FIT trial. BMC Cancer 2012, 12:577.

11. Li JY, Zhang YF, Smith GS, Xue CJ, Luo YN, Chen WH, Skinner CJ, Finkelstein $\mathrm{J}$ : Quality of reporting of randomized clinical trials in Tai Chi interventions-a systematic review. Evid Based Complement Alternat Med 2011, 2011:383245

12. Wang CC, Schmid CH, Rones R, Kalish R, Yinh J, Goldenberg DL, Lee Y, McAlindon T: A randomized trial of tai chi for fibromyalgia. N Engl J Med 2010, 363:743-754.

13. Li F, Harmer P, Fitzgerald K, Eckstrom E, Stock R, Galver J, Maddalozzo G, Batya SS: Tai chi and postural stability in patients with Parkinson's disease. N Engl J Med 2012, 366:511-519.

14. Taylor RE, Haskell WL: Tai Chi exercise and stroke rehabilitation. Top Stroke Rehabil 2007, 14:9-22.

15. Lan C, Chen SY, Lai JS, Wong AMK: Tai Chi Chuan in medicine and health promotion. Evid Based Complement Alternat Med 2013, 2013:502131.

16. Taylor-Piliae RE, Coull BM: Community-based Yang-style Tai Chi is safe and feasible in chronic stroke: a pilot study. Clin Rehabil 2012, 26:121-131.

17. Taylor-Piliae RE, Hoke TM, Hepworth JT, Latt LD, Najafi B, Coull BM: Effect of Tai Chi on physical function, fall rates and quality of life among older stroke survivors. Arch Phys Med Rehabil 2014, 95:816-824.

18. Au-Yeung SS, Hui-Chan CW, Tang JC: Short-form Tai Chi improves standing balance of people with chronic stroke. Neurorehabil Neural Repair 2009, 23:515-522.

19. Julious SA: Sample size of 12 per group rule of thumb for a pilot study. Pharm Stat 2005, 4:287-291.

20. Johanson GA, Brooks GP: Initial scale development: sample size for pilot studies. Educ Psychol Meas 2010, 70:394-400.

21. Sullivan KJ, Tilson JK, Cen SY, Rose DK, Hershberg J, Correa A, Gallichio J, McLeod M, Moore C, Wu SS, Duncan PW: Fugl-Meyer assessment of sensorimotor function after stroke: standardized training procedure for clinical practice and clinical trials. Stroke 2011, 42:427-432.

22. Gladstone DJ, Danells CJ, Black SE: The Fugl-Meyer assessment of motor recovery after stroke: a critical review of its measurement properties. Neurorehabil Neural Repair 2002, 16:232-240.

23. Duffy L, Gajree S, Langhorne P, Stott DJ, Quinn TJ: Reliability (inter-rater agreement) of the Barthel Index for assessment of stroke survivors: systematic review and meta-analysis. Stroke 2013, 44:462-468.

24. Quinn TJ, Langhorne P, Stott DJ: Barthel Index for stroke trials development, properties, and application. Stroke 2011, 42:1146-1151.

25. Major MJ, Fatone S, Roth EJ: Validity and reliability of the Berg Balance Scale for community-dwelling persons with lower-limb amputation. Arch Phys Med Rehabil 2013, 94:2194-2202.

26. Zwick D, Rochelle A, Choksi A, Domowicz J: Evaluation and treatment of balance in the elderly: a review of the efficacy of the Berg Balance Test and Tai Chi Quan. Neurorehabilitation 2000, 15:49-56.

27. Blum L, Korner-Bitensky N: Usefulness of the Berg Balance Scale in stroke rehabilitation: a systematic review. Phys Ther 2008, 88:559-566.

28. Low D, Dixon S: Footscan pressure insoles: accuracy and reliability of force and pressure measurements in running. Gait Posture 2010, 32:664-666.

29. Qiu X, Tian DH, Han CL, Chen W, Wang ZJ, Mu ZY, Li X, Liu KZ: Risk factors correlated with plantar pressure in Chinese patients with type 2 diabetes. Diabetes Technol Ther 2013, 15:1025-1032.
30. Franklyn-Miller A, Bilzon J, Wilson C, McCrory P: Can RSscan footscan D3D ${ }^{\mathrm{TM}}$ software predict injury in a military population following plantar pressure assessment? A prospective cohort study. Foot 2014, 24:6-10.

31. Williams LS, Weinberger M, Harris LE, Clark DO, Biller J: Development of a stroke-specific quality of life scale. Stroke 1999, 30:1362-1369.

32. Goldstein LB, Samsa GP: Reliability of the National Institutes of Health Stroke Scale: extension to non-neurologists in the context of a clinical trial. Stroke 1997, 28:307-310.

33. Hilz MJ, Moeller S, Akhundova A, Marthol H, Pauli E, De Fina P, Schwab S: High NIHSS values predict impairment of cardiovascular autonomic control. Stroke 2011, 42:1528-1533.

34. Pereira S, Graham J, Shahabaz A, Salter K, Foley N, Meyer M, Teasell R: Rehabilitation of individuals with severe stroke: synthesis of best evidence and challenges in implementation. Top Stroke Rehabil 2012, 19:122-131.

35. Zhang Y, Jin H, Ma D, Fu Y, Xie Y, Li Z, Zou Y: Efficacy of integrated rehabilitation techniques of traditional Chinese medicine for ischemic stroke: a randomized controlled trial. Am J Chin Med 2013, 41:971-981.

doi:10.1186/1745-6215-15-484

Cite this article as: Zhang et al:: Applying Tai Chi as a rehabilitation program for stroke patients in the recovery phase: study protocol for a randomized controlled trial. Trials 2014 15:484.

\section{Submit your next manuscript to BioMed Central and take full advantage of:}

- Convenient online submission

- Thorough peer review

- No space constraints or color figure charges

- Immediate publication on acceptance

- Inclusion in PubMed, CAS, Scopus and Google Scholar

- Research which is freely available for redistribution

Submit your manuscript at www.biomedcentral.com/submit
C) Biomed Central 